\title{
Superconductivity from Undressing
}

\author{
J. E. Hirsch \\ Department of Physics, University of California, San Diego \\ La Jolla, CA 92093-0319
}

(November 1, 2018)

\begin{abstract}
Photoemission experiments in high $T_{c}$ cuprates indicate that quasiparticles are heavily 'dressed' in the normal state, particularly in the low doping regime. Furthermore these experiments show that a gradual undressing occurs both in the normal state as the system is doped and the carrier concentration increases, as well as at fixed carrier concentration as the temperature is lowered and the system becomes superconducting. A similar picture can be inferred from optical experiments. It is argued that these experiments can be simply understood with the single assumption that the quasiparticle dressing is a function of the local carrier concentration. Microscopic Hamiltonians describing this physics are discussed. The undressing process manifests itself in both the oneparticle and two-particle Green's functions, hence leads to observable consequences in photoemission and optical experiments respectively. An essential consequence of this phenomenology is that the microscopic Hamiltonians describing it break electron-hole symmetry: these Hamiltonians predict that superconductivity will only occur for carriers with hole-like character, as proposed in the theory of hole superconductivity.
\end{abstract}

\section{INTRODUCTION}

Photoemission experiments in high $T_{c}$ cuprates show that the spectral function has two contributions: a coherent quasiparticle peak, and a broad incoherent background. Recently, Ding et al [1] have provided an insightful discussion of the phenomenology observed in photoemission in terms of the one-electron coherence factor, or quasiparticle weight, Z. The analysis of Ding et al, as well as a variety of other analysis and experimental data 2 , 化, suggest that the quasiparticle coherence increases in high $T_{c}$ cuprates both as the carrier concentration increases in the normal state, as well as as the temperature decreases and the system becomes superconducting.

These observations can be simply understood by assuming that the quasiparticle dressing in high $T_{c}$ cuprates is a decreasing function of the 'local' carrier concentration, more specifically of the probability of finding another carrier in the vicinity of a given carrier. This local carrier concentration will increase both as the total carrier concentration increases through doping, as well as in a dilute carrier concentration regime when Cooper pairs form and the superconducting state develops. This physics is qualitatively depicted in Figure 1.

We argue that the essential physics of the high $T_{c}$ phenomenon is undressing 5.6 . At low carrier density, carriers are heavily dressed in the normal state, due to coupling to a bosonic degree of freedom. When carriers pair and the system becomes superconducting, carriers partially undress. Similarly, when the system is doped in the normal state carriers increasingly undress. This will occur if the coupling to the boson degree of freedom is a function of the local carrier concentration, and becomes weaker as the local carrier concentration increases. This feature, we propose, is what makes the material a high temperature superconductor: carriers will pair in order to undress, i.e. to reduce the coupling to this boson degree of freedom. Paradoxically, by becoming bound in Cooper pairs, carriers become more free. At high carrier concentrations, carriers are undressed already in the normal state and hence superconductivity does not occur.

The undressing will give rise to a lowering of the system's free energy, and hence to the condensation energy of the superconductor. Because it is an undressing transition, it is the kinetic energy that is lowered as the system becomes superconducting [7]: as carriers undress, their effective mass decreases, and this higher mobility in the superconducting state is what provides the'glue' for the collective order. Naturally, this will cause observable manifestations in optical properties [8.9.

There are two key distinct questions related to the understanding of high $T_{c}$ superconductivity. One relates to the nature of the bosonic degree of freedom coupled to the electrons that gives rise to pairing and other phenomena: is it magnetic, electronic, phononic, or other? What is its spectral density? The second question is, what is the essential physics of the phenomenon, and what are the key experimental signatures? These two questions are not necessarily tightly coupled, and here we wish to draw a sharp distinction between them. We do have definite views on the first question, namely that the mechanism is purely electronic [10] rather than magnetic, phonon, or a combination of 
various. However this paper mainly focuses on the second question, for which we propose the undressing scenario. It is possible that this scenario may be applicable independent of the detailed answer to the first question.

However, we argue also that the theory of hole superconductivity 11, 12 naturally leads to the phenomenology described above, and, conversely also, that the phenomenology described above naturally leads to the theory of hole superconductivity. The electron-hole symmetry breaking leads to the undressing scenario, and, the assumption of increased undressing with increased carrier concentration leads to electron-hole symmetry breaking. Furthermore, of the two possible choices, we argue that it is hole carriers that are heavily dressed in the normal state, and that electron carriers instead are lightly dressed, or undressed. As holes pair, or as the system is doped with holes, holes become more 'like' electrons, and they undress. We are not aware of any other microscopic scenario that can describe the physics of 'undressing'. Through the framework of the theory of hole superconductivity it is seen that another unavoidable consequence of this 'undressing' physics is the prediction of tunneling asymmetry of universal sign [13], as well as of charge imbalance in the superconducting state [14].

The microscopic physics is easily described qualitatively: there are carriers in a band, and there is a background degree of freedom at each atom, or site. Presence of a carrier at a site will modify, or 'disrupt', the background degree of freedom. So far, the physics is the same as in a variety of electron-boson models. The essential distinguishing feature here is that the disruption caused by the first carrier on a site is assumed to be different to that caused by a second carrier on that site. This essential feature leads to superconductivity through undressing, with distinct characteristics.

We have proposed in the past a variety of microscopic Hamiltonians to describe this physics: (1) a purely electronic model with two orbitals per site [15]; (2) a model of electrons (one orbital per site) coupled to a spin $1 / 2$ degree of freedom at each site [10,11, and (3) a variety of generalized Holstein models [16 18]. The essential physics of all these Hamiltonians (in the parameter range considered) is the same, qualitatively discussed above. We believe that either of these Hamiltonians is a plausible choice for the description of the physics of electrons in atoms with several electrons in the outer shell, which we believe is the relevant physics: the 'background' degree of freedom is simply a way of representing the excitations of these other electrons. However, such Hamiltonians could also be used to describe other microscopic physics, for example coupling to a high frequency phonon.

Some of the consequences of the physics of undressing for optical properties have already been discussed [5]. In particular, it led to the prediction of apparent optical sum rule violation [], which was recently detected experimentally [8]. This is due to the fact that undressing manifest itself in the two-particle Green's function. However, as discussed in this paper, undressing also manifests itself in the one-particle Green's function. Hence, it will have observable consequences for photoemission experiments. The close connection between the effects observed in photoemission and in optical experiments has recently been emphasized by Norman and coworkers [19.

For most of this paper we will use a specific generalized Holstein model [17, 18 to describe the physics of superconductivity through undressing. The reason is simply that a large amount of theoretical work has been done on the Holstein model. In the Holstein model usually considered [16], the electron-hole symmetric version, the physics of undressing is absent. Nevertheless, much of the mathematical treatment carries over to the generalized model considered here.

Furthermore, we restrict ourselves to the small polaron regime of the model, both because the physics in that regime is most transparent, and because we believe it is the most appropiate regime to describe high $T_{c}$ cuprates. Alexandrov and coworkers [20,21] have performed a large amount of very interesting theoretical work on the symmetric Holstein model in the small polaron regime, and we will draw on some of their seminal contributions. In the conclusion we will however discuss the fundamental difference in the physics described by our model and that described by Alexandrov's work.

\section{THE PHYSICS AND HAMILTONIANS}

The physics is qualitatively depicted in Figure 2. A local bosonic degree of freedom couples to the electron (or hole) at the site. The first electron at the site causes a small distortion of the boson degree of freedom, the second causes a much larger distortion. Conversely, the first hole at the site causes a large change of the boson degree of freedom, the second a small one. The discussion can be consistently carried out with electrons or with holes; we will use the language of holes in most of this paper only because it is somewhat simpler to describe the physics of a few holes rather than that of a lot of electrons. Notice that the key physics of electron-hole asymmetry is introduced at the outset.

Let $\mid n>$ be the ground state of the local boson degree of freedom when there are $\mathrm{n}$ holes at the site, and $\mid n^{l}>$ the l-th excited state of that boson degree of freedom, hence $|n\rangle \equiv \mid n^{0}>$. Consider the ground state overlaps of the boson degree of freedom with different number of holes at the site: 


$$
\begin{aligned}
& S=<0 \mid 1> \\
& T=<1 \mid 2>
\end{aligned}
$$

The physics of undressing arises if $S \neq T$ [5]. Specifically, for holes to undress as the hole concentration increases,

$$
S<T
$$

is required, that is, the overlap matrix element of the ground state of the boson degree of freedom with zero and one hole is smaller than the one between one hole and two holes. The effect will be strongest when $S<<T$; in an ideal situation, one will have $T \sim 1$. The factors $S^{2}$ and $T^{2}$ are the quasiparticle weights of single hole carriers and single electron carriers respectively in a Fermi liquid description: hole carriers will have a small quasiparticle spectral weight, electron carriers a large quasiparticle weight.

When a hole at site $\mathrm{i}$ is destroyed, with the boson degree of freedom in its ground state, one of two things can occur: the boson degree of freedom may make a transition to the ground state with one fewer hole, or end up in an excited state. The first process is a diagonal transition, the second a non-diagonal one. If the site is singly occupied we have, with $c_{i \sigma}$ a hole destruction operator,

$$
c_{i \uparrow}|\uparrow>| 1>=|0>| 1>=|0>| 0>S+\sum_{l \neq 0}|0>| 0^{l}><0^{l} \mid 1>
$$

so that $\mathrm{S}$ gives the probability amplitude for the diagonal transition. Here, the site state is represented as a direct product of the hole occupation state and the boson state. The first term in Eq. (3) conserves energy, preserves the phase of the wave function and gives rise to a coherent process, associated with the quasiparticle contribution to the spectral function; the second part gives the incoherent contribution. Similarly, when a hole is destroyed at a site that is occupied by two holes,

$$
c_{i \uparrow}|\uparrow \downarrow>| 2>=|\downarrow>| 2>=|\downarrow>| 1>T+\sum_{l \neq 0}|\downarrow>| 1^{l}><1^{l} \mid 2>
$$

and the weight of the coherent process is given by $\mathrm{T}$, which is assumed to be larger than S. Hence, coherence will increase as the number of doubly occupied sites (by holes) increases, which will be the case both when hole doping and when hole pairing occurs. The completeness relations

$$
\sum_{l}\left|n^{l}><n^{l}\right|=1
$$

ensure that an increase in the weight of coherent processes is accompanied by a decrease in the weight of incoherent processes, and the spectral function sum rule is satisfied.

Similarly, the amplitudes of the ground state to ground state transitions determine the effective mass of the carriers. If $\mathrm{t}$ is the hopping amplitude for a carrier in the absence of coupling to the boson degree of freedom, the hopping amplitude for a single hole when there are no other holes in either of the two sites involved in the hopping process is

$$
t_{2}=t S^{2}
$$

when there is one other hole it is

$$
t_{1}=t S T \equiv t_{2}+\Delta t
$$

and when there are two other holes it is

$$
t_{0}=t T^{2}
$$

the latter being of course also the hopping amplitude for a single electron when there are no other electrons at the two sites involved. The hopping amplitudes are schematically shown in Fig. 3. Single hole carriers are most heavily dressed and hence have a large effective mass $m_{h}^{*}$, related to the hopping amplitude $t_{2}$ by

$$
m_{h}^{*}=\frac{\hbar^{2}}{2 t_{2} a^{2}}
$$


with $a$ the lattice spacing. When holes hop in the presence of other holes, they do so with hopping amplitude $t_{1}>t_{2}$, as a partial undressing occurs. The effective hopping Hamiltonian arising from these transitions is

$$
\begin{gathered}
H_{h o p}=-\sum_{<i, j>, \sigma} t_{i j}^{\sigma}\left(c_{i \sigma}^{\dagger} c_{j \sigma}+h . c .\right) \\
t_{i j}^{\sigma}=t\left[S^{2}+S(T-S)\left(n_{i,-\sigma}+n_{j,-\sigma}\right)+(T-S)^{2} n_{i,-\sigma} n_{j,-\sigma}\right]
\end{gathered}
$$

The third term in Eq. (10b) will be negligible in the low hole concentration regime for reasonable values of on-site repulsion. Hence the effective Hamiltonian, including also an on-site and nearest neighbor Coulomb repulsion, is

$$
H_{e f f}=-\sum_{<i, j>, \sigma>}\left[t_{2}+\Delta t\left(n_{i,-\sigma}+n_{j,-\sigma}\right)\right]\left(c_{i \sigma}^{\dagger} c_{j \sigma}+h . c .\right)+U \sum_{i} n_{i \uparrow} n_{i \downarrow}+V \sum_{<i j>} n_{i} n_{j}
$$

The difference in hopping amplitudes

$$
\Delta t=t_{1}-t_{2}=t S(T-S)
$$

gives rise to pairing and superconductivity [12]. The undressing and accompanying effective mass lowering when pairing occurs gives rise to apparent violation of the optical sum rule as discussed in detail elsewhere [7,5].

Furthermore, in the normal state the effective hopping for a hole will be an increasing function of hole concentration $n$ [12], given by

$$
t(n)=t_{2}+n \Delta t
$$

This will cause an expansion of the effective bandwidth as the hole concentration increases, as shown schematically in Fig. 4. The conductivity sum rule yields for the integrated optical absorption for intra-band processes

$$
\int_{0}^{\omega_{m}} d \omega \sigma_{1}(\omega)=\frac{\pi e^{2} n}{2 m^{*}}
$$

with $\sigma_{1}$ the frequency-dependent real part of the conductivity (per site), $n$ the number of carriers per site, $m^{*}$ the effective mass and $\omega_{m}$ a high frequency cutoff that allows only for intra-band processes. Using Eqs. (9) and (13),

$$
\int_{0}^{\omega_{m}} d \omega \sigma_{1}(\omega)=\frac{\pi e^{2} a^{2} t(n) n}{\hbar^{2}}
$$

so that the integrated low frequency spectral weight increases faster than linearly with carrier concentration. There is evidence from optical experiments that the low frequency optical spectral weight increases with doping more rapidly than expected from the added number of carriers [22], in support of Eq. (15). This transfer of optical spectral weight from high to low frequencies with hole doping is a manifestation of the undressing that occurs with increasing hole concentration, and will be accompanied by a decrease in the spectral weight of nondiagonal transitions, i.e. hopping processes where the background degrees of freedom end up in excited states rather than the ground state.

The relation between quasiparticle spectral weight and effective mass discussed above follows of course from general properties of many-body systems. The exact Green's function for a many-body system can be written as

$$
G(k, \omega)=\frac{1}{\omega-\epsilon_{k}-\Sigma(k, \omega)}
$$

where $\Sigma$ is the self-energy, and $\epsilon_{k}$ is measured from the chemical potential. In the models considered here the self-energy has no $k$-dependence, and we have for its real part

$$
\Sigma_{r e}(\omega)=\Sigma_{r e}(0)+\omega \frac{\partial \Sigma_{r e}}{\partial \omega}
$$

$\Sigma_{r e}(0)$ just renormalizes the chemical potential. Hence

$$
G(k, \omega)=\frac{1}{\omega\left(1-\frac{\partial \Sigma_{r e}}{\partial \omega}\right)-\epsilon_{k}}+G^{\prime}=\frac{Z}{\omega-Z \epsilon_{k}}+G^{\prime}
$$


with

$$
Z=\frac{1}{1-\frac{\partial \Sigma_{r e}}{\partial \omega}}
$$

The term $G^{\prime}$ contains the imaginary part of the self-energy and gives rise to the incoherent contribution. Eq. (18) shows that the same factor $Z$, the wave function renormalization factor that results from the frequency dependence of the real part of the self-energy, determines the quasiparticle spectral weight and the effective mass renormalization. In the models considered here, $Z$ is a function of carrier concentration and we have simply

$$
Z(n)=S^{2}+n S(T-S)+\frac{n^{2}}{4}(T-S)^{2}
$$

in the normal state, with $n$ the hole concentration per site. In particular $Z=S^{2}$ or $Z=T^{2}$ for an almost filled band $(n \rightarrow 0)$ or an almost empty band $(n \rightarrow 2)$ respectively. In the superconducting state, $Z$ will increase as the pair amplitude develops.

One Hamiltonian that describes this physics is the spin-fermion model [10,11] with site Hamiltonian given by

$$
H_{i}=\left(V\left(n_{i \uparrow}+n_{i \downarrow}\right)-\omega_{0}\right) \sigma_{z}^{i}+\Delta \sigma_{x}^{i}+U n_{i \uparrow} n_{i \downarrow}
$$

with $\sigma^{i}$ a spin $1 / 2$ degree of freedom, and $n_{i \sigma}$ a hole occupation operator. The physics described here arises in the parameter regime $V>\omega_{0}, \Delta<<\omega_{0}$. Then, the spin degree of freedom points approximately up when there is no hole at the site, and approximately down when there are one or two holes at the site, as shown schematically in Fig. 3. The change in the spin state is much larger between hole occupation 0 and 1 than between hole occupation 1 and 2 , hence $S<<T$.

Another Hamiltonian that describes this physics is an electronic model with two orbitals per site [15], and a site Hamiltonian

$$
H_{i}=U n_{i \uparrow} n_{i \downarrow}+U^{\prime} n_{i \uparrow}^{\prime} n_{i \downarrow}^{\prime}+V n_{i} n_{i}^{\prime}+\epsilon n_{i}^{\prime}-t^{\prime}\left(c_{i \sigma}^{\dagger} c_{i \sigma}^{\prime}+\text { h.c. }\right)
$$

where the primed and unprimed operators refer to electrons in the two site orbitals. The physics of undressing will arise if the condition

$$
U^{\prime}+2 \epsilon<V-\epsilon<U
$$

is satisfied, together with the ordering $U, U^{\prime}, V>>\epsilon>>t^{\prime}$. These conditions ensure that a single electron resides primarily in the lower level (unprimed orbital) while two electrons reside dominantly in the higher level (primed orbitals). When an electron leaves a doubly occupied orbital, the second electron relaxes to the lower energy level giving a large renormalization effect, and $S<<1$, while $T=1$. This Hamiltonian in the parameter regime described may be justified from first principles atomic physics calculations [23].

Finally, the physics described here will arise in a variety of generalizations of the Holstein Hamiltonian [18] describing electrons interacting with a local displacement degree of freedom $q_{i}$

$$
H_{i}=\frac{p_{i}}{2 m\left(n_{i}\right)}+\frac{1}{2} K\left(n_{i}\right) q_{i}^{2}+\alpha\left(n_{i}\right) q_{i} n_{i}+U n_{i \uparrow} n_{i \downarrow} .
$$

The undressing effect arises when the usual Holstein model is generalized by allowing for an occupation dependence of the parameters, $\mathrm{m}, \mathrm{K}$ or $\alpha$ in Eq. (24), or by adding an anharmonic term $\beta q^{4}$ to the site Hamiltonian. In the remainder of this paper we will use one particular version of this model, where the coupling $\alpha$ depends on site occupation.

\section{A GENERALIZED HOLSTEIN MODEL}

We consider the site Hamiltonian

$$
H_{i}=\frac{p^{2}}{2 m}+\frac{1}{2} K q^{2}+q\left[\alpha\left(n_{\uparrow}+n_{\downarrow}\right)-\alpha^{\prime} n_{\uparrow} n_{\downarrow}\right]+U n_{\uparrow} n_{\downarrow}
$$

where the site index on the right-hand side is understood. The new term in this Hamiltonian, proportional to $\alpha^{\prime}$, breaks electron-hole symmetry and gives rise to the physics of undressing. This term may be understood as arising from a dependence of the electron-boson coupling on the hole occupation $\left(\alpha\left(n_{i}\right)=\alpha-\alpha^{\prime}\left(n_{\uparrow}+n_{\downarrow}-1\right) / 2\right)$. Assuming 
$\alpha, \alpha^{\prime}>0$, Eq. (25) implies that the electron-boson coupling becomes weaker as holes are added. Alternatively, the new term may be understood as a modification of the on-site Coulomb repulsion by the boson displacement, $U(q)=U-\alpha^{\prime} q$. In terms of boson creation and annihilation operators the Hamiltonian is

$$
H=\hbar \omega\left(a^{\dagger} a+\frac{1}{2}\right)+M\left(a^{\dagger}+a\right)\left[n_{\uparrow}+n_{\downarrow}-\frac{\alpha^{\prime}}{\alpha} n_{\uparrow} n_{\downarrow}\right]+U n_{\uparrow} n_{\downarrow}
$$

with $M=\alpha\left(\hbar \omega_{0} / 2 K\right)^{1 / 2}, \omega_{0}=(K / m)^{1 / 2}$. Our Hamiltonian differs from the usual Frohlich-type Hamiltonian in that the boson couples to a term that is quartic in fermion operators in addition to the usual quadratic one. [24]

By completing the squares in Eq. (25), we obtain the effective on-site repulsion

$$
U_{e f f}=U-\frac{2 \alpha^{2}+\alpha^{\prime 2}-4 \alpha \alpha^{\prime}}{2 K}
$$

We assume $\alpha^{\prime} \leq \alpha$. Note that $\alpha^{\prime}$ causes an increase in the on-site repulsion; in particular, for $\alpha^{\prime}>(2-\sqrt{2}) \alpha$, the reduction in $U$ due to the electron-boson coupling $\alpha$ is completely offset, and $U_{\text {eff }}>U$. Still, the model in that regime will give rise to superconductivity due to the undressing effect produced by $\alpha^{\prime}$. The polaron site energy is given by

$$
\epsilon_{0}=-\frac{\alpha^{2}}{K}
$$

The equilibrium position of the oscillators with $n$ holes at the sites, $q_{n}$, is

$$
\begin{gathered}
q_{0}=0 \\
q_{1}=-\frac{\alpha}{K} \\
q_{2}=-\frac{2 \alpha}{K}+\frac{\alpha^{\prime}}{K}
\end{gathered}
$$

and the ground state overlap matrix elements for the states with $n$ and $n^{\prime}$ holes is

$$
<n \mid n^{\prime}>=e^{-\frac{K}{4 \hbar \omega_{0}}\left(q_{n}-q_{n^{\prime}}\right)^{2}}
$$

so that

$$
\begin{gathered}
S=e^{-\frac{\alpha^{2}}{4 K \hbar \omega_{0}}} \equiv e^{-\frac{g^{2}}{2}} \\
T=e^{-\frac{\left(\alpha-\alpha^{\prime}\right)^{2}}{4 K \hbar \omega_{0}}} \equiv e^{-\frac{g^{2}}{2}(1-\gamma)^{2}}
\end{gathered}
$$

with

$$
\begin{gathered}
g=\frac{\alpha}{\sqrt{2 K \hbar \omega_{0}}}=\frac{M}{\hbar \omega_{0}} \\
\gamma=\frac{\alpha^{\prime}}{\alpha}
\end{gathered}
$$

Hence, $T>S$, and in particular $T=1$ for $\alpha^{\prime}=\alpha$. For that particular case, $U_{\text {eff }}=U+\alpha^{2} / 2 K$. The effective low energy Hamiltonian to first order in the bare hopping $t$ is

$$
H_{e f f}=-\sum_{(i, j)}\left(t_{2}+\Delta t\left(n_{i,-\sigma}+n_{j,-\sigma}\right)\right)\left(c_{i \sigma}^{\dagger} c_{j \sigma}+h . c .\right)+U_{e f f} \sum_{i} n_{i \uparrow} n_{i \downarrow}
$$

with $t_{2}$ and $\Delta t$ given by Eqs (6) and (12). Superconductivity will occur if the condition 12] 


$$
\frac{T}{S}>\left(1+\frac{U_{\text {eff }}}{D_{h}}\right)^{1 / 2}
$$

is satisfied, with $D_{h}=2 z t_{2}$ the single hole bandwidth ( $\mathrm{z}$ is the number of nearest neighbors to a site). Hence for $T \sim 1$ and sufficiently small $S$, superconductivity will occur for arbitrarily large values of the on-site repulsion $U_{\text {eff }}$. Some discussion of the effect of a nearest neighbor Coulomb repulsion and of higher order corrections to the parameters in $H_{e f f}$ is given in ref. 18. Matrix elements between ground state oscillator states and excited states with different occupation number are given by

$$
\begin{gathered}
<0\left|1^{l}>=<1\right| 0^{l}>=\frac{e^{-g^{2} / 2} g^{l}}{(l !)^{1 / 2}} \\
<1\left|2^{l}>=<2\right| 1^{l}>=\frac{e^{-g^{2}(1-\gamma)^{2} / 2}(g(1-\gamma))^{l}}{(l !)^{1 / 2}}
\end{gathered}
$$

To calculate the single particle Green's function and spectral function it is useful to formulate the problem in terms of polaron operators, as is done in the usual Holstein model. In the absence of hopping between sites, the Hamiltonian is diagonalized by a generalized Lang-Firsov transformation [25],6]

$$
\bar{H}_{i}=e^{G} H_{i} e^{-G}
$$

with

$$
G=g\left(a^{\dagger}-a\right)\left(n_{\uparrow}+n_{\downarrow}-\gamma n_{\uparrow} n_{\downarrow}\right)
$$

with $a^{\dagger}$ a boson creation operator

$$
a^{\dagger}=\frac{1}{\sqrt{2}}\left(\sqrt{\frac{m \omega_{0}}{\hbar}} q+i \frac{1}{\sqrt{m \hbar \omega_{0}}} p\right)
$$

Boson operators transform according to

$$
\bar{a}=e^{G} a e^{-G}=a-g\left(n_{\uparrow}+n_{\downarrow}-\gamma n_{\uparrow} n_{\downarrow}\right)
$$

and fermion operators transform according to

$$
\begin{aligned}
& \bar{c}_{i \sigma}=e^{G} c_{i \sigma} e^{-G}=c_{i \sigma} X_{i \sigma} \\
& X_{i \sigma}=e^{-g\left(a^{\dagger}-a\right)\left(1-\gamma n_{i,-\sigma}\right)}
\end{aligned}
$$

In contrast to the usual Lang-Firsov transformation, the "dressing" operators $X_{i \sigma}$ here depend of fermion occupation number. The hopping part of the Hamiltonian

$$
H_{h o p}=-t \sum_{(i, j)}\left(c_{i \sigma}^{\dagger} c_{j \sigma}+\text { h.c. }\right)
$$

becomes

$$
\bar{H}_{h o p}=-t \sum_{(i, j)}\left(X_{i \sigma}^{\dagger} X_{j \sigma} c_{i \sigma}^{\dagger} c_{j \sigma}+h . c .\right)
$$

The expectation value of the $\mathrm{X}$-operators in the zero boson subspace is

$$
<X_{i \sigma}>=e^{-\frac{g^{2}}{2}\left(1-\gamma n_{i,-\sigma}\right)^{2}}
$$

i.e. the overlap matrix elements $S$ or $T$ of Eq. (31) depending on the value of the hole occupation $n_{i,-\sigma}$. 


\section{EXACT RESULTS}

Consider a system with a single site. The single particle spectral function at zero temperature for holes with spin up is given by

$$
A_{n_{\uparrow} n_{\downarrow}}^{\uparrow}(\omega)=2 \pi \sum_{l}\left[|<l| c_{\uparrow}^{\dagger}|0>|^{2} \delta\left(w-\left(E_{l}^{(n+1)}-E_{0}^{n}\right)\right)+|<l| c_{\uparrow}|0>|^{2} \delta\left(w+\left(E_{l}^{(n-1)}-E_{0}^{n}\right)\right)\right]
$$

where $\mid 0>$ is the ground state of the system with $n_{\sigma}$ holes of $\operatorname{spin} \sigma$, and $\mid l>$ are states of the system with $n_{\uparrow}+1$ or $n_{\uparrow}-1$ holes with spin up. Using Eq. (3) we obtain

$$
A_{n_{\uparrow} 0}^{\uparrow}(\omega)=2 \pi S^{2} \delta\left(\omega-\epsilon_{0}\right)+2 \pi \sum_{l \neq 0}\left|<1^{l}\right| 0>\left.\right|^{2}\left[\left(1-n_{\uparrow}\right) \delta\left(w-\epsilon_{0}-\omega_{l}\right)+n_{\uparrow} \delta\left(w-\epsilon_{0}+\omega_{l}\right)\right]
$$

with $\epsilon_{0}$ the site energy for one hole and $\omega_{l}$ the energy of the l-th excited state of the boson. In a many body system the spectral function has the form [26]

$$
A(k, \omega)=2 \pi Z(k) \delta\left(\omega-\left(E_{k}-\mu\right)\right)+A_{\text {inc }}(k, \omega)
$$

that is, a sharp $\delta$-function describing the quasiparticle and an incoherent background $A_{\text {inc }}$. The weight of the $\delta$ function, $0 \leq Z \leq 1$, gives the degree of coherence. For our case Eq. (45), the first term, corresponding to diagonal transitions, represents the coherent part, and the second term is the incoherent part, hence $Z=S^{2}$. If instead the ground state of the site has a spin down hole the spectral function is

$$
\begin{aligned}
A_{n_{\uparrow} 1}^{\uparrow} & =2 \pi T^{2} \delta\left(\omega-\epsilon_{0}-U_{e f f}\right)+2 \pi \sum_{l \neq 0}\left[\left|<2^{l}\right| 1>\left.\right|^{2}\left[\left(1-n_{\uparrow}\right) \delta\left(w-\epsilon_{0}-U_{e f f}-\omega_{l}\right)\right.\right. \\
& \left.+n_{\uparrow} \delta\left(w-\epsilon_{0}-U_{e f f}+\omega_{l}\right)\right]
\end{aligned}
$$

where $\left(2 \epsilon_{0}+U_{\text {eff }}\right)$ is the energy of two holes at the site. Here, the quasiparticle weight is $Z=T^{2}$. We assumed the operators in Eq. (44) to be hole operators, and $S<T$. Then, as holes are added to the site the coherent part of the spectral function increases and the incoherent part decreases, so that the sum rule

$$
\int d \omega A_{n}(\omega)=2 \pi
$$

is satisfied.

For the case of the generalized Holstein model defined in the previous section, $\omega_{l}=l \omega_{0}$ and

$$
\begin{aligned}
A_{n_{\uparrow} 0}^{\uparrow}(\omega)= & 2 \pi e^{-g^{2}} \delta\left(\omega-\epsilon_{0}\right)+2 \pi e^{-g^{2}} \sum_{l=1}^{\infty} \frac{g^{2 l}}{l !}\left[\left(1-n_{\uparrow}\right) \delta\left(w-\epsilon_{0}-l \omega_{0}\right)+n_{\uparrow} \delta\left(w-\epsilon_{0}+l \omega_{0}\right)\right] \\
A_{n_{\uparrow} 1}^{\uparrow}(\omega)= & 2 \pi e^{-g^{2}(1-\gamma)^{2}} \delta\left(\omega-\epsilon_{0}-U_{e f f}\right)+2 \pi e^{-g^{2}(1-\gamma)^{2}} \times \\
& \sum_{l \neq 0}\left[\frac{g^{2 l}(1-\gamma)^{l}}{l !}\left[\left(1-n_{\uparrow}\right) \delta\left(w-\epsilon_{0}-U_{e f f}-l \omega_{0}\right)+n_{\uparrow} \delta\left(w-\epsilon_{0}-U_{e f f}+l \omega_{0}\right)\right]\right.
\end{aligned}
$$

The qualitative behavior is shown in Fig. 5. Similar qualitative behavior is found for the two other models discussed in Sect. II.

This simple example bears directly on the understanding of qualitative features of photoemission experiments in high $T_{c}$ cuprates. When the system has a low concentration of holes in the normal state, the spectral function will look qualitatively like Fig. (5a). The $l=0$ peak, which gives the quasiparticle contribution, will be nearly absent if $g^{2}$ is large. The other peaks in an extended system will merge into a continuum incoherent contribution, peaking at some high energy. Both when the system is doped with holes, and at fixed doping when the temperature is lowered and superconductivity sets in, the number of doubly occupied (by holes) sites will increase, and hence the spectral function will have a larger contribution of Fig. 5(b). Hence the intensity of the quasiparticle peak in the spectral function will increase both as the system goes superconducting and as the system is doped in the normal state.

Similarly, consider the optical absorption resulting from transitions between localized particles at sites 1 and 2 . Assume the current operator is given by [26] 


$$
j=\sum_{\sigma} p_{12} c_{2 \sigma}^{\dagger} c_{1 \sigma}+\text { h.c. }
$$

The real part of the optical conductivity at zero temperature is given by

$$
\sigma_{1}(\omega)=\frac{\pi}{\omega} \sum_{m}|<m| j|0>|^{2} \delta\left(\omega-\left(E_{m}-E_{0}\right)\right)
$$

For the case where there is one hole at site 1 and no hole at site 2 in the ground state, Eq. (51) yields

$$
\sigma_{1}^{(1)}(\omega)=\frac{\pi}{\omega} p_{12}^{2}\left[S^{4} \delta(\omega)+\sum_{\left(l l^{\prime}\right) \neq(00)}\left|<0^{l} l 1>\right|^{2}\left|<1^{l^{\prime}}\right| 0>\left.\right|^{2} \delta\left(\omega-\omega_{l}-\omega_{l^{\prime}}\right)\right]
$$

and for the case where there is one hole at each site, of opposite spin

$$
\sigma_{1}^{(2)}(\omega)=\frac{2 \pi}{\omega} p_{12}^{2}\left[S^{2} T^{2} \delta\left(\omega-U_{e f f}\right)+\sum_{\left(l l^{\prime}\right) \neq(00)}\left|<0^{l}\right| 1>\left.\right|^{2}\left|<2^{l^{\prime}}\right| 1>\left.\right|^{2} \delta\left(\omega-U_{e f f}-\omega_{l}-\omega_{l^{\prime}}\right)\right]
$$

Once again we have separated the coherent contribution, involving no excited bosons, from the incoherent contribution where bosons are emitted. For the generalized Holstein model, using the relation

$$
\sum_{l=0}^{L} \sum_{l^{\prime}=0}^{L-l} \frac{g_{1}^{2 l} g_{2}^{2 l^{\prime}}}{l ! l^{\prime} !}=\frac{\left(g_{1}+g_{2}\right)^{L}}{L !}
$$

these relations become

$$
\begin{gathered}
\sigma_{1}^{(1)}(\omega)=\frac{\pi}{\omega} p_{12}^{2}\left[e^{-2 g^{2}} \delta(\omega)+e^{-2 g^{2}} \sum_{l=1}^{\infty} \frac{\left(2 g^{2}\right)^{l}}{l !} \delta\left(\omega-\omega_{0} l\right)\right] \\
\sigma_{1}^{(2)}(\omega)=\frac{2 \pi}{\omega} p_{12}^{2}\left[e^{-g^{2}\left[1+(1-\gamma)^{2}\right]} \delta\left(\omega-U_{e f f}\right)+e^{-g^{2}\left[1+(1-\gamma)^{2}\right]} \sum_{l=1}^{\infty} \frac{g^{2 l}\left[1+(1-\gamma)^{2}\right]^{l}}{l !} \delta\left(\omega-U_{\text {eff }}-\omega_{0} l\right)\right]
\end{gathered}
$$

The qualitative behavior of these quantities is similar to that of the single particle spectral functions shown in Fig. 5 . In an extended system the zero boson terms give rise to the intra-band optical absorption. According to these results, for $\gamma \neq 0$ the intra-band absorption will increase as holes are added to the system more rapidly than proportional to the number of holes, since there is an increasing contribution of $\sigma_{1}^{(2)}(\omega)$ relative to $\sigma_{1}^{(1)}(\omega)$. Correspondingly, the contribution of $l \neq 0$ terms, corresponding to non-intra-band processes, decreases. The two other models discussed in section II display similar physics [5].

Furthermore, as the system with a dilute concentration of holes becomes superconducting, the relative contribution of $\sigma_{1}^{(2)}$ will also increase relative to that of $\sigma_{1}^{(1)}$, because there is an increased fraction of configurations with holes on the same or on nearest neighbor sites. In that case the resulting extra intra-band optical spectral weight goes into the $\delta$-function that determines the London penetration depth, and an apparent violation of the Ferrell-Glover-Tinkham conductivity sum rule results. [7]5]

In summary, in the two simple examples discussed in this section it is seen that the process of undressing will manifest itself similarly in the one-particle and two-particle spectral functions, which determine the results of photoemission and optical absorption measurements. For both types of observables, within the class of models discussed here, an increase in the local hole concentration through doping or through pairing gives rise to increased coherence.

\section{SPECTRAL FUNCTION IN THE DILUTE LIMIT}

To obtain further insight on the effect of undressing in connection with superconductivity we consider the dilute limit, that is, the limit when the number of hole carriers in the band goes to zero. The wavefunction for a single pair of holes governed by the effective Hamiltonian Eq. (11) can be found exactly and is of the form 27]

$$
\left|\Psi>=\frac{1}{\sqrt{N}} \sum_{k} f_{k} c_{k \uparrow}^{\dagger} c_{-k \downarrow}^{\dagger}\right| 0>=\frac{1}{\sqrt{N}} \sum_{i, j} f(i-j) c_{i \uparrow}^{\dagger} c_{j \downarrow}^{\dagger} \mid 0>
$$




$$
f_{k}=\sum_{j} e^{i R_{j}} f(j)
$$

with

$$
\sum_{\delta}|f(\delta)|^{2}=1
$$

Equations that determine the amplitudes $f(\delta)$ and the pair binding energy $E_{b}$ are found from exact solution of the Schrodinger equation for a single pair [27]. In particular, in the limit where $t_{2} \rightarrow 0$ in Eq. (11) only the amplitudes $f(\delta)$ for on-site and nearest neighbor pairs are nonvanishing [28].

We consider the part of the spectral function Eq. (44) corresponding to the destruction of a hole of momentum $\mathrm{k}$. Applying the operator

$$
c_{k \uparrow}=\frac{1}{\sqrt{N}} \sum_{k} e^{i k R_{i}} c_{i \uparrow}
$$

to the wavefunction Eq. (56a) gives rise to diagonal and non-diagonal terms, as given by Eqs. (3) and (4). The diagonal terms, where the bosons remain in the ground state, yields

$$
\left(c_{k \uparrow} \mid \Psi>\right)_{\operatorname{diag}}=\frac{1}{\sqrt{N}}\left[(T-S) f(0)+S f_{k}\right] c_{-k \downarrow} \mid 0>
$$

and the non-diagonal terms are

$$
\begin{aligned}
\left(c_{k \uparrow} \mid \Psi>\right)_{\text {non-diag }} & =\frac{1}{N} \sum_{i, l} e^{i k R_{i}} f(0)\left|\downarrow>{ }_{i}<1^{l}\right| 2> \\
& +\frac{1}{N} \sum_{i \neq j, l} e^{i k R_{i}} f(i-j)\left|\downarrow>_{j}\right| 0^{l}>_{i}<0^{l} \mid 1>
\end{aligned}
$$

Assuming that states with excited bosons in a real space representation are approximately eigenstates of the Hamiltonian one obtains the spectral function

$$
\begin{aligned}
A_{s}(k, \omega) & =2 \pi n_{p}\left[\left[(T-S) f(0)+S f_{k}\right]^{2} \delta\left(\omega+\epsilon_{k}+E_{b}\right)\right. \\
& \left.+\sum_{l \neq 0}\left[\left|<1^{l} 2>\right|^{2} f^{2}(0)+\left|<0^{l}\right| 1>\left.\right|^{2}\left(1-f(0)^{2}\right)\right] \delta\left(\omega+\epsilon_{k}+E_{b}+\omega_{l}\right)\right]
\end{aligned}
$$

We have assumed that there are $n_{p}$ pairs in the ground state that don't interfere with one another. In contrast, in the standard models with no electron-hole symmetry breaking such as the conventional Holstein model, $S=T$ and Eq. (60) becomes

$$
A_{s}(k, \omega)=2 \pi n_{p}\left[S^{2} f_{k}^{2} \delta\left(\omega+E_{b}\right)+\sum_{l \neq 0}\left|<0^{l}\right| 1>\left.\right|^{2} \delta\left(\omega+\epsilon_{k}+E_{b}+\omega_{l}\right)\right]
$$

Comparing Eqs. (60) and (61) shows clearly the effect of electron-hole symmetry breaking. The coherent part of the spectral function in the superconducting state will be enhanced when $T>S$ proportionally to the on-site pair wave function amplitude $f(0)$. As a consequence the enhancement will be larger the smaller the coherence length, i.e. the size of the pair wave function. Correspondingly, the incoherent part of the spectral function is reduced, since $\left|<1^{l}\right| 2>|<|<0^{l}|1>|$ if $S<T$, as discussed in Sect. II. Furthermore, the enhancement is proportional to the number of pairs. The number of pairs within a two-fluid model is given by the superfluid density, $n_{p}=\lambda_{L}(0)^{2} / \lambda_{l}(T)^{2}$, with $\lambda_{L}$ the London penetration depth, and grows proportionally to the gap squared as the temperature is lowered below $T_{c}$. Also, the superfluid density will increase with doping. Hence in the presence of the undressing effect we conclude that there will be an extra contribution to the coherent part of the spectral function that increases both as the temperature is lowered and superconductivity sets in, and as the doping is increased in the superconducting state. These effects should be observable in photoemission experiments. We discuss these effects in more detail after considering the spectral function in the normal state in the next section. 


\section{SPECTRAL FUNCTION IN THE NORMAL STATE}

The calculation of the spectral function in the normal state of the generalized Holstein model follows closely the treatment of Alexandrov and Ranninger for the electron-hole symmetric case [21]. In the single particle Green's function

$$
G(m, \tau)=-<T_{\tau} c_{0 \uparrow}(\tau) c_{m \uparrow}^{\dagger}(0)>
$$

fermion operators are replaced by the transformed operators Eq. (35), and averages over fermions and bosons are decoupled. The result for the spectral function is

$$
\begin{aligned}
A(k, \omega)= & 2 \pi e^{-g^{2}}\left[\left[1+n\left(e^{\gamma g^{2}}-1\right)\right] \delta\left(\omega+\left(\epsilon_{k}-\mu\right)\right)\right. \\
+ & \frac{1}{N} \sum_{k^{\prime}} \sum_{l=1}^{\infty} \frac{g^{2 l}}{l !}\left[1+n\left[e^{\gamma g^{2}}(1-\gamma)^{l}-1\right]\right] \times \\
& {\left[\left(1-n_{k^{\prime}}\right) \delta\left(\omega+l \omega_{0}+\epsilon_{k^{\prime}}-\mu\right)+n_{k^{\prime}} \delta\left(\omega-l \omega_{0}+\epsilon_{k^{\prime}}-\mu\right)\right] }
\end{aligned}
$$

where $n$ is the hole concentration. For $\gamma=0$ it reduces to the result of Alexandrov and Ranninger. The first term describes the coherent contribution, the second term the incoherent one. When $\gamma>0$, the coherent part increases at the expense of the incoherent part as the hole concentration $n$ increases. The amplitude of the coherent part of the spectral function is proportional to the effective bandwidth, which is given (to lowest order in $\gamma$ ) by

$$
D(n)=2 z t(n)=2 z\left(t_{2}+n \Delta t\right)=2 z t e^{-g^{2}}\left(1+n\left(e^{\gamma g^{2}}-1\right)\right)
$$

and increases linearly with doping.

In a photoemission experiment the quantity probed is

$$
I_{0}(k, \omega)=A(k, \omega) f(\omega)
$$

with $f(\omega)$ the Fermi function. To take into account experimental resolution we calculate the convolution

$$
I(k, \omega)=\int d \omega^{\prime} F\left(\omega-\omega^{\prime}\right) I_{0}\left(k, \omega^{\prime}\right)
$$

with $F(\omega)$ a Gaussian function with width $\sigma_{\omega}$. An example of the behavior obtained is shown in Fig. 6 . Note that the quasiparticle peak is absent for low hole concentration and appears as the hole concentration increases.

\section{QUASIPARTICLE SPECTRAL WEIGHT}

Here we summarize the qualitative conclusions on the quasiparticle spectral weight that we can draw from the results of the previous two sections. In the normal state, the quasiparticle weight $\mathrm{Z}$ is, from Eqs. (63) and (46)

$$
Z_{n}=S^{2}\left(1+n\left(\frac{T}{S}-1\right)\right)=e^{-g^{2}}\left(1+n\left(e^{\gamma g^{2}}-1\right)\right)
$$

The first form is general for the class of models discussed in section II, the second the particular case of the generalized Holstein model. In the superconducting state the quasiparticle weight, from Eq. (60), is

$$
Z_{s}=n_{p}\left[(T-S) f(0)+S f_{k}\right]^{2}
$$

For the particular case $T>>S$ and the generalized Holstein model,

$$
Z_{s}=n_{p} e^{-g^{2}}\left(e^{\gamma g^{2}}-1\right)^{2} f^{2}(0)
$$

Equations (67) and (69) show that the quasiparticle weight will grow both as the system is doped in the normal state and as the system goes into the superconducting state. However, because the factor $\left(e^{\gamma g^{2}}-1\right)$ appears squared in the expression for $Z_{s}$, the onset of coherence will be more pronounced as the temperature is lowered than as $n$ is increased in the normal state if that factor is large, which will be the case for $S<<1$ and $T>>S$. This appears to be the observation in photoemission experiments [1]. More generally, the conditions $S<<1$ and $T>>S$ are 
consistent with the observations of a high degree of incoherence in the underdoped regime of cuprates and onset of coherence for a relatively modest increase in $n$. Furthermore, in the model of hole superconductivity that condition, which is equivalent to $\Delta t>>t_{2}$, and $t_{2}$ very small, is required to fit a variety of experiments [12].

In that case, one may expect to see a pronounced quasiparticle peak emerge as superconductivity sets in even in the underdoped regime. The temperature dependence of $Z_{s}$ is governed by $n_{p}$, the superfluid weight, which is what is observed experimentally [1]. The amplitude of the pair wave function $f(0)$ is found to be essentially independent of temperature in the model of hole superconductivity [12.

Furthermore, the doping dependence of the quasiparticle peak in the superconducting state Eq. (69) will be determined by that of the superfluid weight $n_{p}$, and also by the doping dependence of $f(0)$. In the model of hole superconductivity the superfluid weight is found to grow approximately linearly with doping [7]. The on-site pair amplitude $f(0)$ is found to decrease as the system moves towards the overdoped regime because there is a cross-over to the weak coupling regime and the coherence length increases [12,29]. Ding et al [1] find $\mathrm{Z}$ in the superconducting state to increase linearly with doping in the underdoped regime, and taper off on the overdoped side. Hence this behavior is qualitatively consistent with the prediction of Eq. (69). Detailed quantitative comparison will be given elsewhere.

Finally, the expression Eq. (67) for the quasiparticle weight in the normal state predicts that a quasiparticle peak should be seen in the normal state for sufficiently high doping, which is also consistent with observations [1].

\section{CONCLUSIONS}

We have considered in this paper a class of models that appear to contain key ingredients of the phenomenology inferred from a wide range of photoemission and optical experiments in high $T_{c}$ cuprates. This phenomenology is that increased coherence is observed both in the normal state when the hole concentration increases, and for fixed hole concentration when the temperature is reduced and the system goes superconducting. The emergence of coherence thus appears to be associated with an increase in the 'local' carrier concentration. We have pointed out that this phenomenology follows from the class of models considered in the theory of hole superconductivity. In these models, the first hole on a site causes a large disruption in a background degree of freedom, the second hole a much smaller disruption. Electron-hole asymmetry thus results as an essential feature. The resulting low energy effective Hamiltonian contains the term $\Delta t$, which leads to superconductivity and is a manifestation of the undressing process. We illustrated this physics with some simple examples.

An explicit calculation of the single particle spectral function for one such model, a generalized Holstein model, showed that indeed it exhibits a decrease in the incoherent contribution and the emergence of a quasiparticle peak in the normal state as the hole concentration increases. Furthermore, we showed in the particular case of the dilute limit that increased coherence also results in the superconducting state as a result of the formation of the pair wave function. An approximate calculation of the one-particle spectral weight in the superconducting state for a more general case will be discussed in a forthcoming paper.

These models have similar consequences for the two-particle Green's function, and predict that a transfer of optical spectral weight from high to low frequency in the normal state should occur as the hole doping increases due to the undressing process. Furthermore, in the superconducting state the undressing process gives rise to an 'apparent' violation of the Ferrell-Glover-Tinkham low frequency optical sum rule [7],8] accompanied by a decrease in the optical absorption at high frequencies [5.9], signaling the fact that the superconducting condensation energy is kinetic energy.

Other models have been recently discussed in the literature where the superconducting condensation energy is argued to come from kinetic energy [30,31]. However none of those models address the questions of from where, and through which mechanism, the transfer of high frequency optical spectral weight occurs.

Even though the Hamiltonian considered here is related to the model studied by Alexandrov in his theory of bipolaronic superconductivity [20], there are fundamental differences. Alexandrov's model, by not breaking electronhole symmetry, does not contain the physics of undressing. Also, his model predicts pair formation (bipolarons) in the normal state, which subsequently Bose-condense. Instead, in the models discussed here, because of the enhanced pair mobility due to the undressing process, the transition to the superconducting state occurs through pair binding rather than Bose-condensation, except in extremely low carrier concentration regimes [32]. Finally, we believe that the relevant boson excitations that were described here through the generalized Holstein model are likely to be electronic excitations of the atomic shells 10,11,23,33 rather than related to ionic lattice displacements as in Alexandrov's work.

In summary, we believe that the basic physics discussed here is relevant to the understanding of high $T_{c}$ superconductivity in cuprates. Because of its generality it should also apply to any Fermi liquid system where the quasiparticle dressing decreases as the local carrier concentration increases, and we suggest that this may be generally the case for metals for which at least some of the quasiparticles in the normal state have hole-like character. This is also consistent 
with the empirical observation that superconductivity appears to be quite generally associated with positive values of the Hall coefficient in the normal state at least in some directions. 34,35. Thus, the possibility that this physics may play an essential role in all superconductors should not be excluded [36]. Note, however, that the physics of undressing discussed here leads uniquely to the symmetry of the superconducting state being s-wave [12].

\section{ACKNOWLEDGMENTS}

The author is grateful to D. Basov, M. Norman and H. Suhl for stimulating discussions.

[1] H. Ding et al, cond-mat/0006143 and references therein.

[2] T. Takahashi et al, Physica B 165\&166, 1221 (1990).

[3] D.S. Dessau et al, Phys. Rev. Lett. 66, 2160 (1991).

[4] A.V. Puchkov et al, Phys. Rev. B 54, 6686 (1996).

[5] J. E. Hirsch, Physica C 201, 347 (1992).

[6] J. E. Hirsch, in "Polarons and Bipolarons in high- $T_{c}$ Superconductors and Related Materials", ed. by E.K.H. Salje, A.S. Alexandrov and W.Y. Liang, Cambridge University Press, Cambridge, 1995, p. 234.

[7] J. E. Hirsch, Physica C 199, 305 (1992); J.E. Hirsch and F. Marsiglio, Physica C 331, 150 (2000); cond-mat/0004496, submitted to Phys.Rev. B.

[8] D.N. Basov et al, Science 283, 49 (1999); A.S. Katz et al, Phys.Rev. B 61, 5930 (2000); D.N. Basov et al, preprint.

[9] I. Fugol et al, Sol.St.Comm. 86, 385 (1993).

[10] J. E. Hirsch, Phys.Lett. A134, 451 (1989).

[11] J.E. Hirsch and S. Tang, Sol.St.Comm. 69, 987 (1989); Phys. Rev. B 40, 2179 (1989); J.E. Hirsch and F. Marsiglio, Phys. Rev. B 41, 2049 (1990).

[12] J.E. Hirsch and F. Marsiglio, Phys. Rev. B 39, 11515 (1989); Phys. Rev. B 45, 4807 (1992); Physica C 162-164, 591 (1989); F. Marsiglio and J.E. Hirsch, Phys. Rev. B 41, 6435 (1990).

[13] F. Marsiglio and J.E. Hirsch, Physica C 159, 157 (1989); J.E. Hirsch, Phys. Rev. B 59, 11962 (1999).

[14] J.E. Hirsch, Phys. Rev. Lett. 72, 558 (1994); Phys. Rev. B 58, 8727 (1998).

[15] J.E. Hirsch, Phys. Rev. B 43, 11400 (1991).

[16] T. Holstein, Ann. Phys. (N.Y.) 8, 325 (1959).

[17] P. Pincus, Sol. St. Comm.11, 51 (1972).

[18] J.E. Hirsch, Phys. Rev. B 47, 5351 (1993).

[19] M.R. Norman, M. Randeria, B. Janko and J.C. Campuzano, Phys. Rev. B 61, 14742 (2000).

[20] A.S. Alexandrov, Phys. Rev. B 61, 12315 (2000) and references therein.

[21] A.S. Alexandrov and J. Ranninger, Physica C 198, 360 (1992).

[22] S. Uchida et al, Phys. Rev. B 43, 7942 (1991).

[23] J.E. Hirsch, Phys. Rev. B 48, 3340 (1993).

[24] L.J. Sham, Sol.St. Comm. 20, 623 (1976).

[25] I.G. Lang and Y.A. Firsov, Sov.Phys. JETP 16, 1301 (1963).

[26] G.D. Mahan, "Many-Particle Physics", Plenum, New York, 1981.

[27] F. Marsiglio and J. E. Hirsch, Physica C 171, 554 (1990).

[28] J. E. Hirsch, Physica C 161, 185 (1989).

[29] F. Marsiglio and J. E. Hirsch, Physica C 165, 71 (1990).

[30] P.W. Anderson, Science 268, 1154 (1995); S. Chakravarty, H.Y. Young and E. Abrahams, Phys.Rev.Lett. 82, 2366 (1999).

[31] V.J. Emery , S.A. Kivelson and J.M. Tranquada, cond-mat/9907228, to appear in Proc. Natl. Acad. Sci. USA .

[32] J. E. Hirsch, Physica C 179, 317 (1991).

[33] J. E. Hirsch, Chem.Phys.Lett. 171, 161 (1990).

[34] I.M. Chapnik, Phys.Lett. 72A, 255 (1979).

[35] J.E. Hirsch, Phys. Rev. B 55, 9007 (1997).

[36] J. E. Hirsch, Physica C 158, 326 (1989); Phys.Lett. A138, 83 (1989). 
FIG. 1. Phenomenology of high $T_{c}$ superconductors (schematic) as described by the models in this paper. Heavily dressed quasiparticles at low concentrations undress as the temperature is lowered or as the carrier concentration increases. s.t. denotes spectral weight transfer (from high to low frequencies in the direction of the arrows), or strip tease. Below the curve labeled $T_{c}$ the system is superconducting. The initial rise in $T_{c}$ versus $n$ is due to the increasing number of carriers.

FIG. 2. The physics of electron-hole asymmetric polarons. A boson degree of freedom is associated with each site. The first electron at the site causes a small change in the ground state of this degree of freedom, the second electron causes a large change. For holes, the situation is reversed. Two examples of the boson degree of freedom are shown, an oscillator and a spin $1 / 2$.

FIG. 3. Schematic depiction of hopping processes for different band fillings. As the (electron) band filling increases, the hopping amplitude decreases and carriers become heavier. $n_{h}$ denotes hole concentration.

FIG. 4. Electronic band versus doping (schematic) as predicted by Eq. (13). As the hole doping increases, the Fermi level moves down and the band becomes broader.

FIG. 5. Spectral function for a site for the generalized Holstein model. The lowest peak gives the quasiparticle weight, the other peaks give the incoherent contribution which in an extended system will broaden into a smooth spectrum, given by the envelope dashed lines. (a) corresponds to Eq. (44a), with $n_{\downarrow}=0$, and (b) to Eq. (44b) with $n_{\downarrow}=1$. As the hole concentration increases the weight of the quasiparticle (q.p.) peak increases and the incoherent contribution shifts to lower frequencies and decreases in total weight. Parameters used are $g^{2}=3, \gamma=0.5$.

FIG. 6. Angle resolved photoemission spectrum for low (solid lines) and high (dashed lines) hole concentrations, from Eq. (61). For each case two values of the momentum are shown, $\epsilon_{k}-\mu=10 \mathrm{meV}$ and $\epsilon_{k}-\mu=20 \mathrm{meV}$. Parameters used are $g^{2}=4, \gamma=0.75, \omega_{0}=10 \mathrm{meV}, \sigma_{\omega}=10 \mathrm{meV}$. 


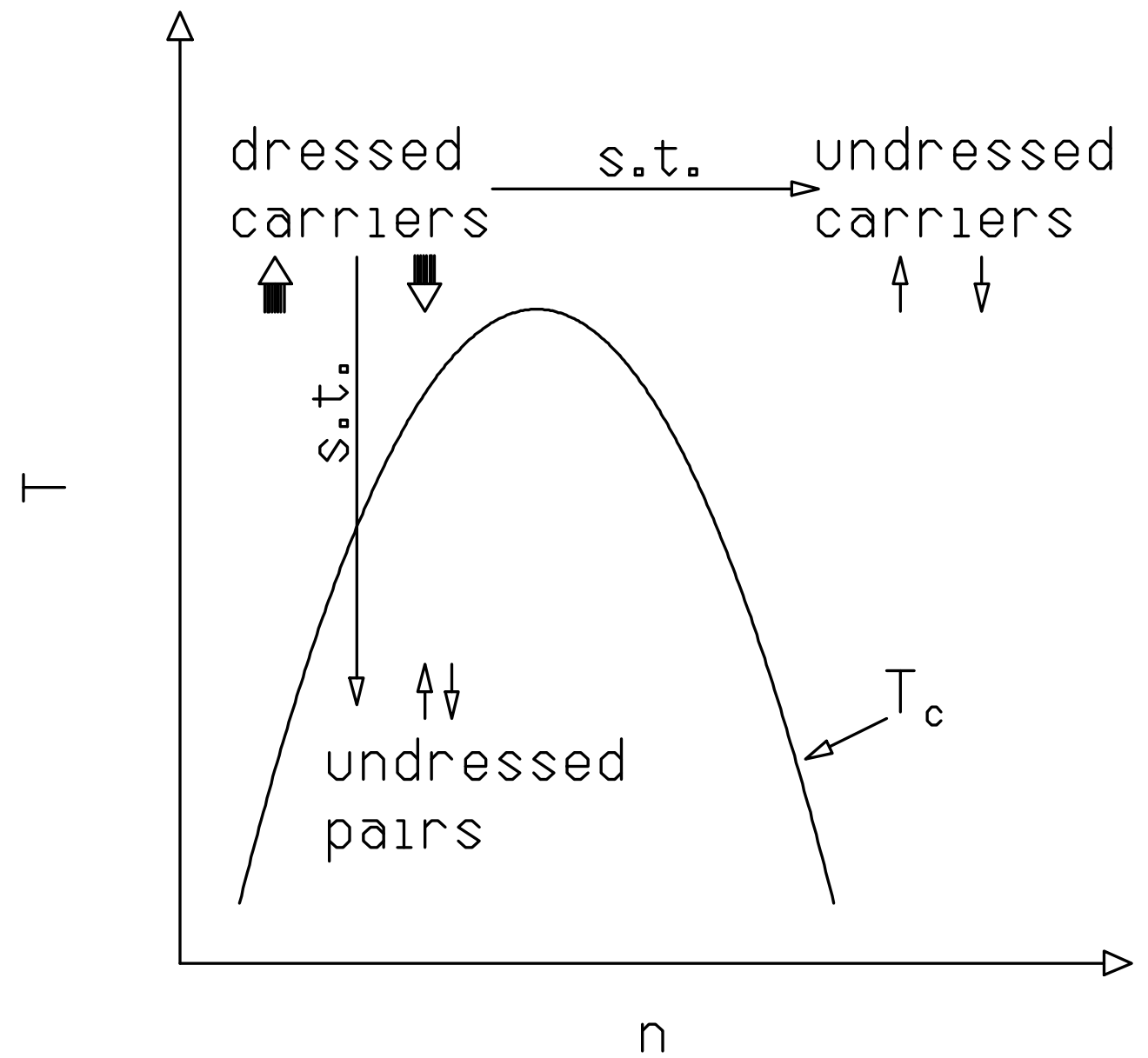




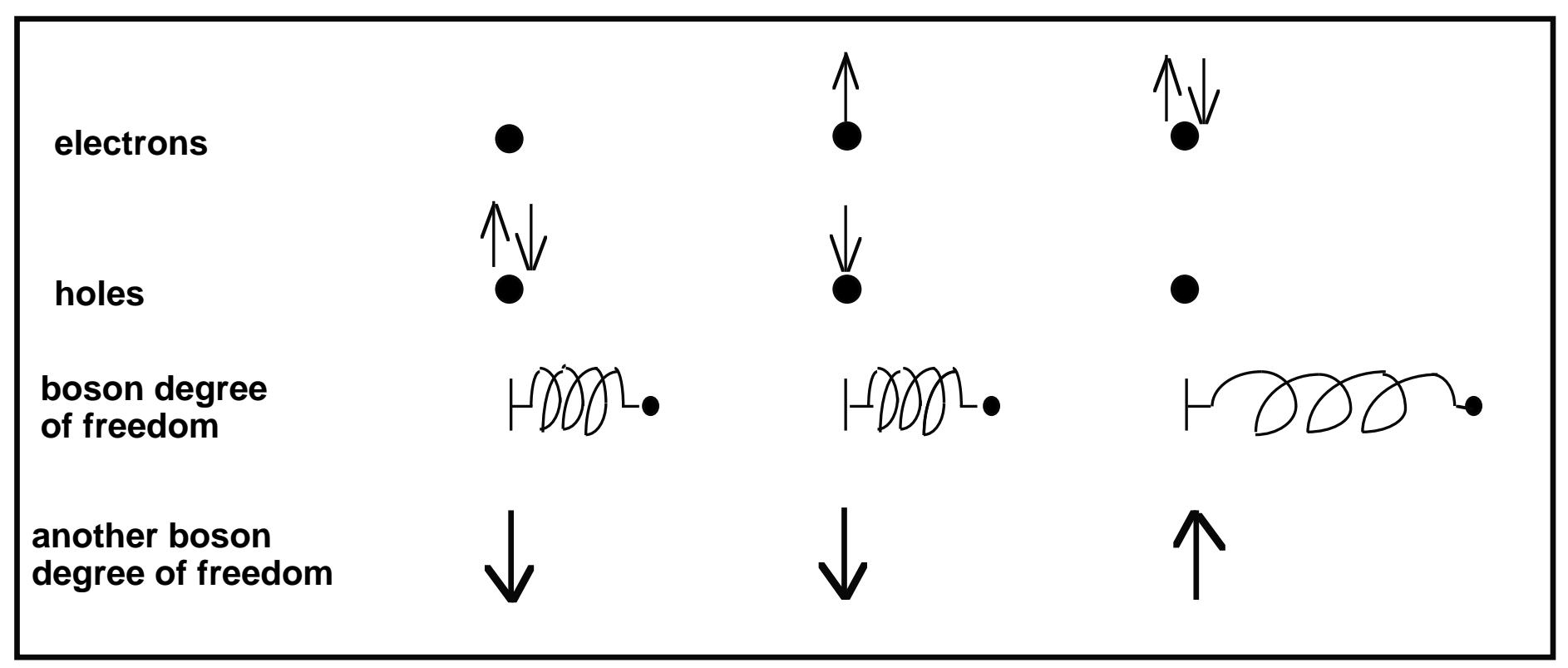




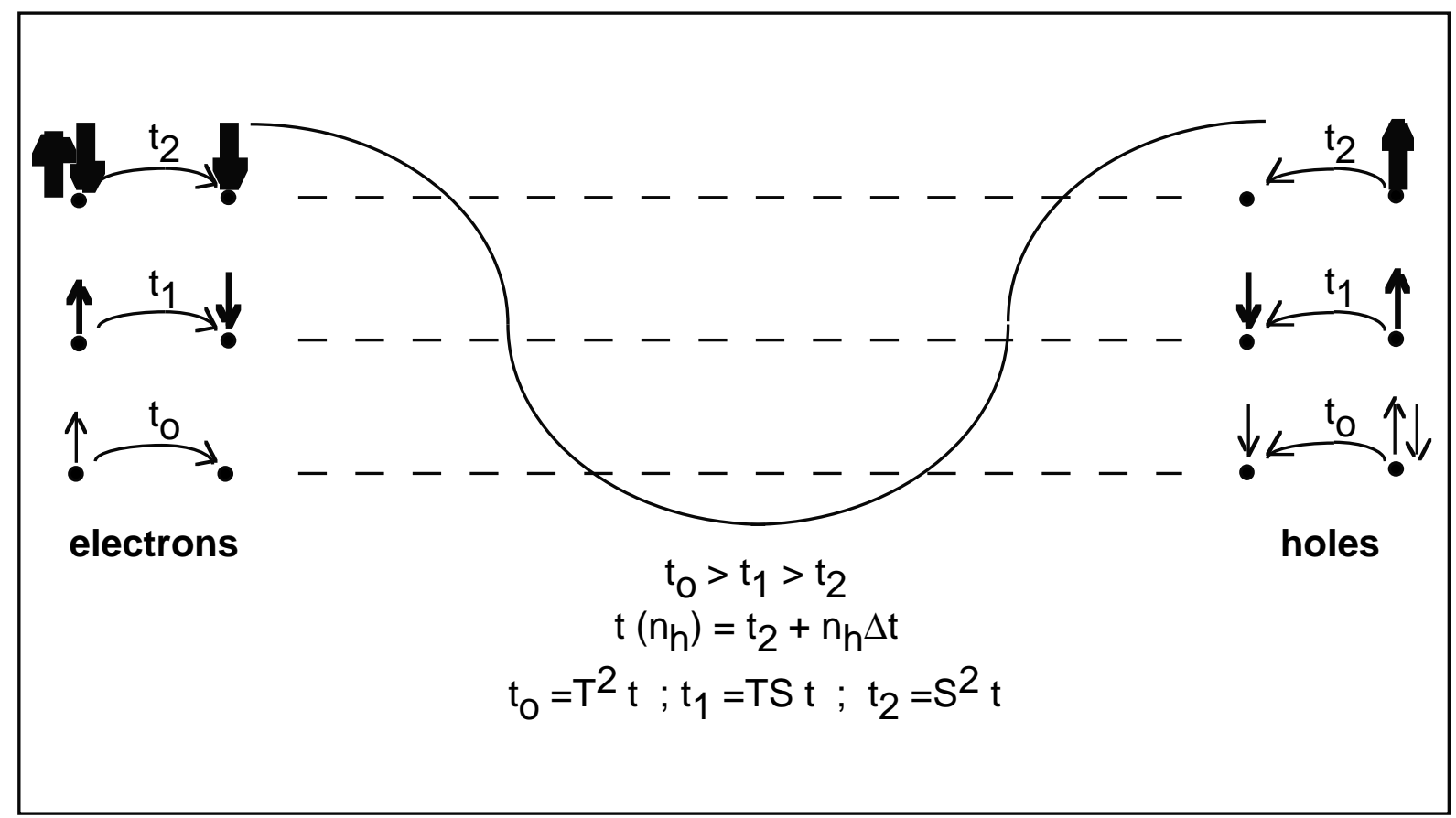




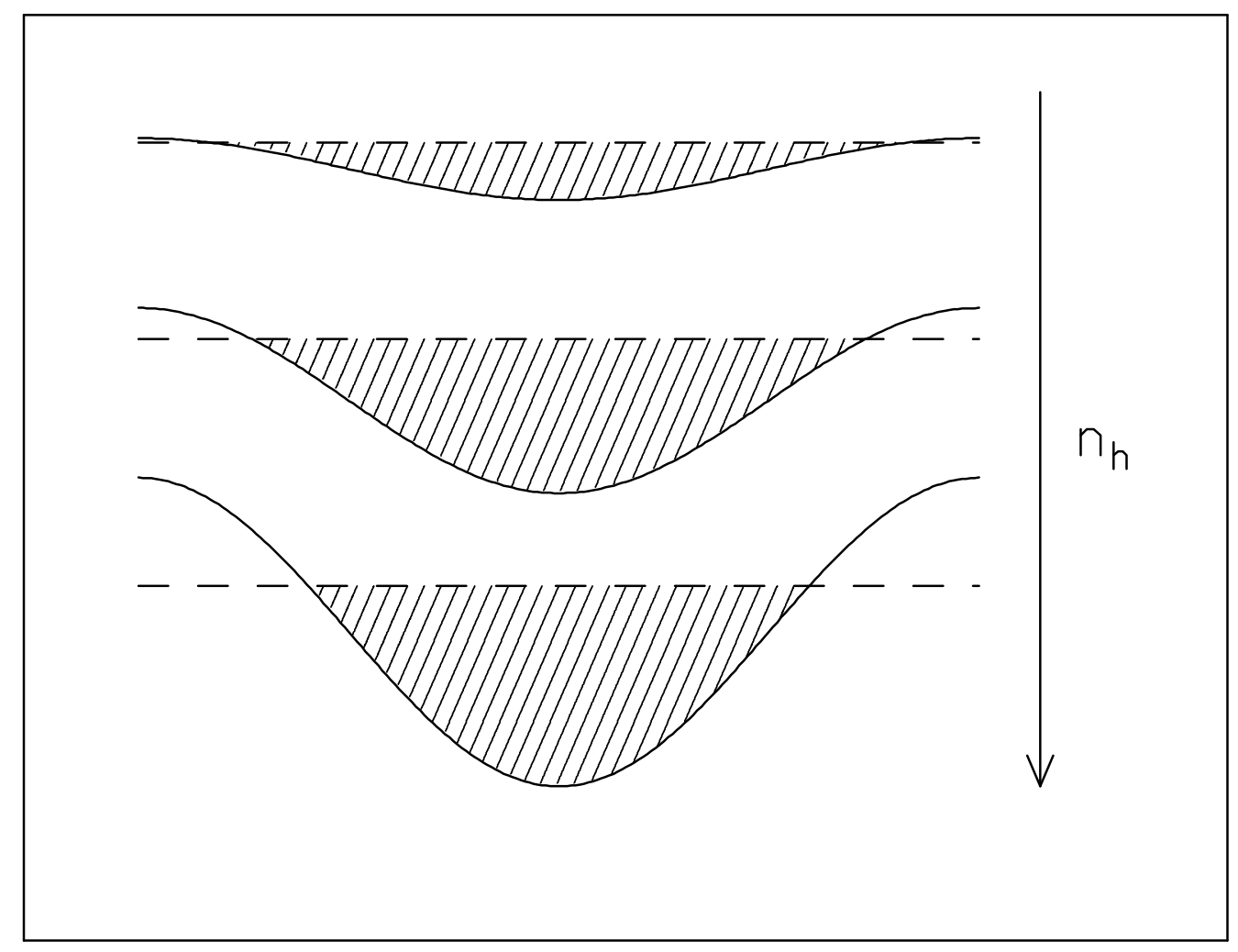




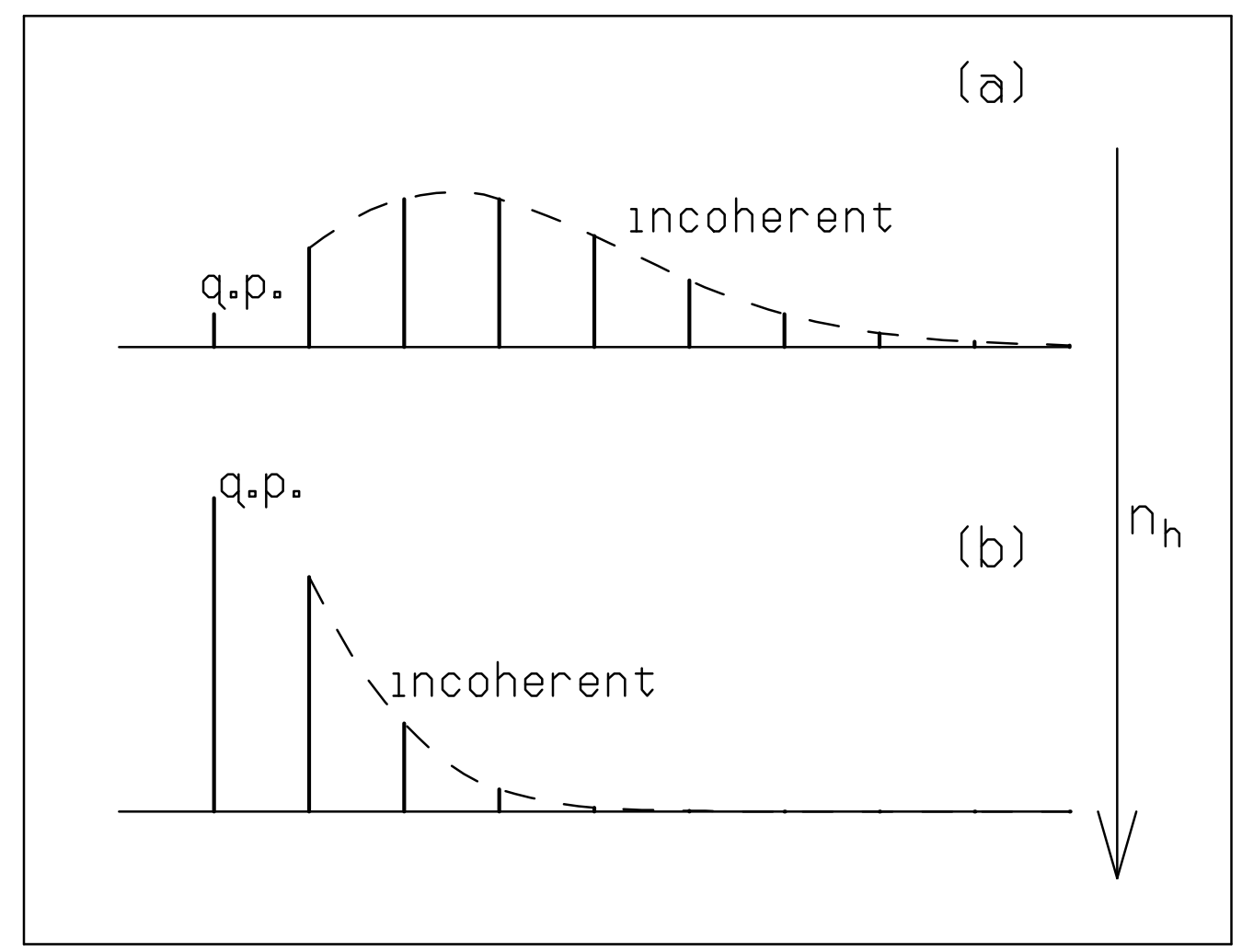




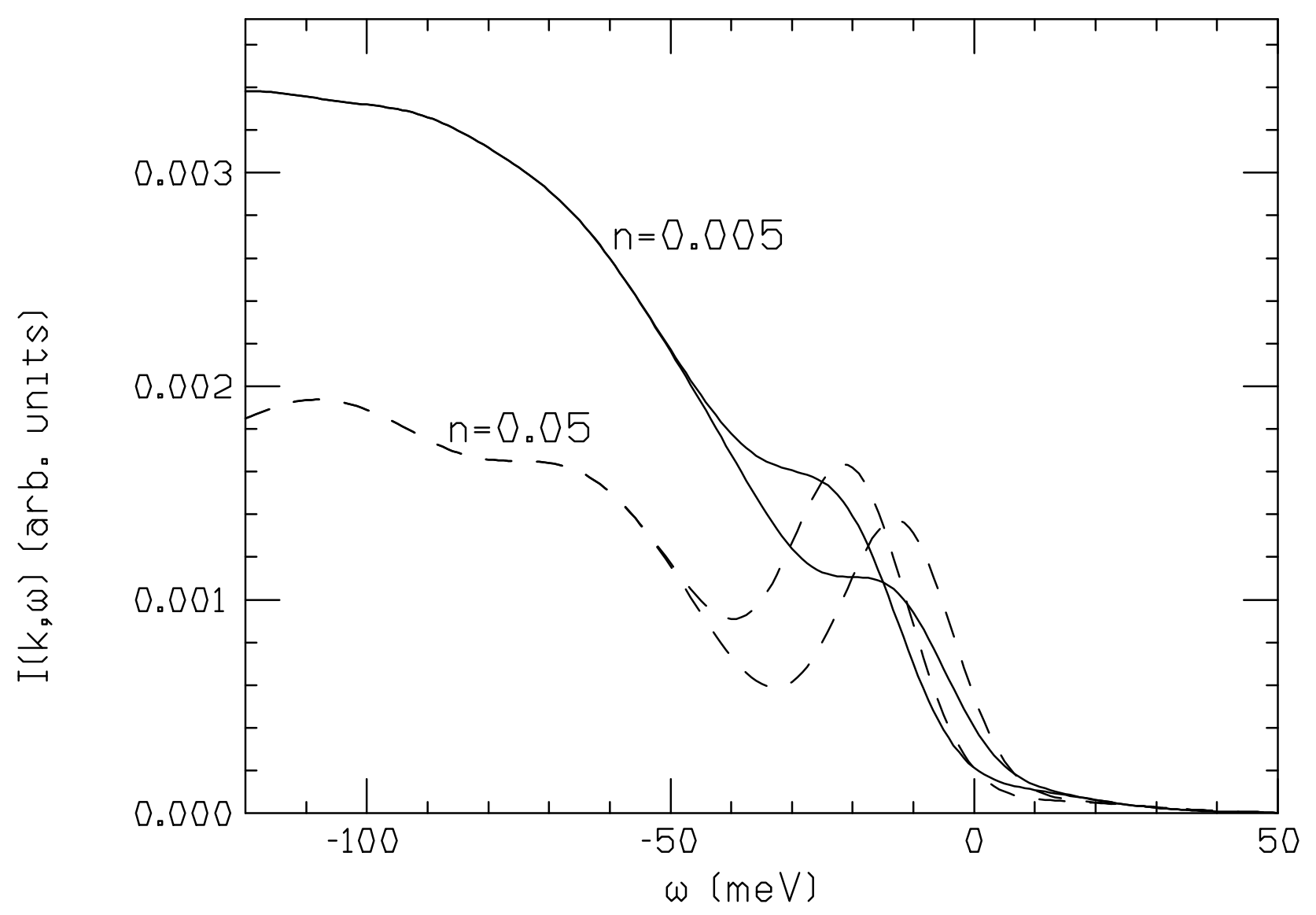

